\title{
Dynamic Response of Gravity Dam Model using Isogeometric Analysis
}

\author{
Rodnny Jesus Mendoza \\ Fakhye \\ Federal Technological \\ University of Paraná (UTFPR), \\ Brazil
}

\author{
Geraldo Carvalho Brito \\ Junior \\ State University of West \\ Paraná (UNIOESTE), Brazil
}

\author{
Roberto Dalledone \\ Machado \\ Federal University of Paraná \\ (UFPR), Brazil
}

\begin{abstract}
Gravity dams are structures commonly used in electricity generation, water supply, flood control and other purposes. Concerns about the safety of these structures when subjected to dynamic loads are an important issue for new projects and in maintenance programs for already built dams. Isogeometric Analysis (IGA) is a numerical approach that allows the discretization and analysis of continuous medium using the approximation functions generated in the construction of digital models or Computer Aided Design (CAD) models. In the present study, first IGA is applied in the study of free vibration behavior of a two dimensional dam model. Then the dynamic response of the structure subjected to time varying loads is obtained using the Central Difference Method (CDM). Numerical tests are performed to show the applicability and future applications are discussed.
\end{abstract}

\section{Keywords}

Dynamic response, Isogeometric analysis, Gravity dam.

\section{INTRODUCTION}

Gravity dams are structures commonly used in electricity generation, water supply, flood control and other purposes. A gravity dam is essentially a solid concrete structure that resists imposed forces principally by its own weight and they are the most common of the concrete types and the simplest type to design and build [1]. The dynamic analysis of a dam is an essential phase for both in assessing the safety of existing dams and to evaluate proposed projects for new dams. This dynamic analysis mostly consists in obtain the dynamic response of the structure subjected to external time dependent excitations.

Structural Health Monitoring (SHM) [2] considers the analysis of vibrations in dams as a fundamental problem for the design and construction of dams. Excessive vibrations may generate damage, defined as changes in the material, geometric shapes, including changes in boundary conditions or support, which can adversely affect the performance of the structure [3].

In the case of gravity dams, the dynamic behavior of these structures has been studied with some success due to the fact that this type of dam is suitable to be modeled using 2D analysis. The reliability of this procedure has been verified by demonstrating that the expected results are generally consistent with field observations. With the introduction of the Finite Element Method (FEM) and the advances in computational techniques to obtain the dynamic response, the procedures of dam design started to change. In technical literature is possible to find as early as the 70 s, publications presenting dynamic analysis work based on FEM. Those works overcame some shortcomings of the static approach and still there are significant problems to solve [4].
Nowadays, among the numerical methods used in modeling and analysis of structures, the Finite Element Method (FEM) [5]-[7] is still one of the most widely used among engineers and designers.

In the classical formulation of FEM, polynomial interpolation functions are used both in the evaluation of the unknowns as in the geometry approximation. The functions normally employed (Ex. Lagrange Polynomials) are different from those used by CAD software. Cottrell et al. [8] indicates that the generation of local and global finite element matrices can consume up to $60 \%$ of the time used for modeling and analysis. Interpolations made by the finite element basis functions are not integrated with those used in the generation of the solid model. It follows, therefore, that the interpolation process is duplicated, since it occurs in two different ways for the same problem: First, the generation of the CAD model, and second in the matrix generation of the FEM [9].

Isogeometric Analysis (IGA) is a recent approach where the approximation functions are those traditionally used in computer graphics, commonly known as NURBS (Non Uniform Rational B-Splines).

An advantage of this approach is the direct communication between CAD environments and analysis environment, enabling time optimization in pre-processing and analysis phases. Still, IGA allows to work in the "exact" geometry making possible to eliminate imperfections and modeling errors by providing more accurate solutions for certain problems [9].

Cottrell et al. [10] found that IGA shows better dynamic response than conventional finite element formulation in the frequency spectrum. Discontinuities in accuracy appear only in the region of higher frequencies of vibration problems. This feature can be decisive in wave propagation studies.

Considering these properties, the application of IGA on dam vibration problems is an interesting and promising application since the use of CAD environments is an established practice in design of new dams and in the documentation of dams built in pre-digital era.

The use of digital geometric information generated in CAD systems of a dam already built or in design stage, would be a significant contribution avoiding additional modeling steps and will contribute to improving the safety of these structures. Fig. 1 shows tridimensional CAD models of the Itaipu dam in Brazil retrieved from analogical documentation. 


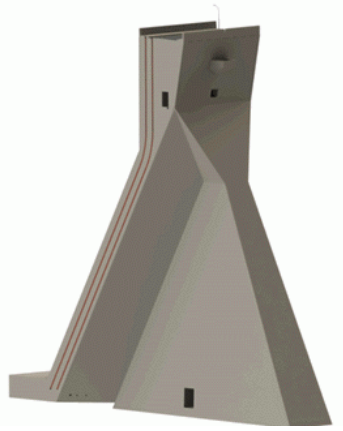

Fig. 1: CAD model of an Itaipu dam section (Brazil) [11]

\section{ISOGEOMETRIC ANALYSIS}

Since the introduction of the basic concepts of IGA by Hughes et al. [9], several studies have been conducted to extend its application to different fields of computational mechanics. In those studies, IGA has shown similar or superior performance compared to conventional FEM, and there was found a precision gain in the treatment of certain problems such as turbulence, nonlinear analysis of shell type structures, shape optimization and aerodynamics [12]-[15].

Finite elements with low order formulations are also found to suffer locking phenomenon in flexural dominated problems. The FEM solution with high order functions would be expensive from the computational point of view. Mathematical characteristics of NURBS functions are expected to give IGA a more efficient performance [8].

\subsection{Fundamentals of IGA}

A mesh of finite elements is a discretization of the domain of analysis, which is divided by several subdomains that are the elements. In the FEM context, because of mapping an element has two representations, one in the parametric domain and the other in the physical domain. The elements are usually defined by their coordinates and nodal degrees of freedom that are usually the values of the basis functions at the nodes. Shape functions is another name for these basis functions [16].

There are two mesh definitions in IGA: the control mesh and the physical mesh. Control points define the control mesh, and the control mesh interpolates the control points. The appearance of the control mesh is similar to a finite element mesh, but there is no direct relationship between them. The unknowns of the problem are the degrees of freedom located at the control points [8].

The relationships between the geometric and parametric domains in an IGA based code are illustrated in Fig. 2. In this figure there are shown: the physical domain, the parametric domain and the domain corresponding to an isogeometric element. The description of the basis functions and the numerical formulation of the problem will be shown below.

NURBS functions are constructed from a set of points in parameter space called knots, normally grouped in vectors. These knot vectors define the geometry of the physical network and they are not necessarily associated to degrees of freedom. In linear space, an element within the IGA is defined as the domain between two separate control points. Similarly, it is possible to generalize this concept to two-dimensional and three-dimensional spaces [16]

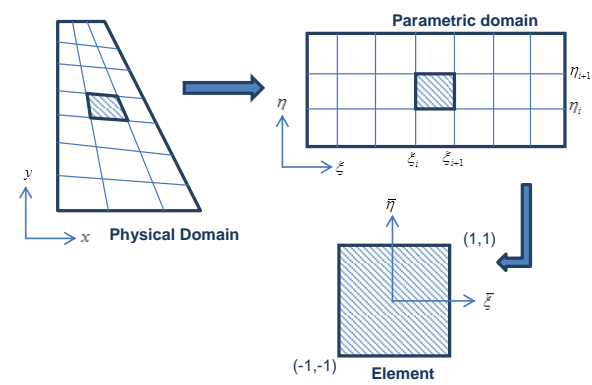

Fig. 2: Different domains and relationships in IGA

\subsection{Basis functions}

The knot vector is a non-decreasing set of coordinates in parameter space given by $\Xi=\left\{\xi_{1}, \xi_{2}, \ldots, \xi_{n+p+1}\right\}$ where $\xi_{i}$ is the $i$-th knot, $p$ is the polynomial degree and $n$ is the number of basis functions used to generate the curve.

Knot vectors can be equally spaced (uniform) or not (nonuniform). A single coordinate of a knot may be repeated taking the same value, this feature is called multiplicity.

Basis functions are directly influenced by knot vectors. An open vector is one in which the control points at the beginning and the end are repeated $p+1$ times. Open vectors are the standard in CAD environments [9].

The basis functions of a B-spline parametric curve are defined recursively. For $p=0$ :

$$
N_{i, 0}=\left\{\begin{array}{l}
1 \text { if } \xi_{i} \leq \xi<\xi_{I+1} \\
0 \text { else }
\end{array}\right.
$$

And for $p=1,2,3 \ldots$ :

$$
N_{i, p}(\xi)=\frac{\xi-\xi_{i}}{\xi_{i+p}-\xi_{i}} N_{i, p-1}(\xi)+\frac{\xi_{i+p+1}-\xi}{\xi_{i+p+1}-\xi_{i+1}} N_{i+1, p-1}(\xi)
$$

The derivatives of the B-spline functions that are necessary for IGA matrices are also defined recursively [17].

\subsection{NURBS}

NURBS curves can be obtained by the projective transformation of a B-spline curve [8]. B-Spline curves are constructed from the linear combination of the basis functions B-Spline and control points. For $n$ basis functions with $n$ control points $\mathbf{P}_{i}$, the polynomial equation of a B-spline curve is given by:

$$
\mathbf{C}(\xi)=\sum_{i=1}^{n} N_{i, p}(\xi) \mathbf{P}_{i}
$$

Given a control mesh $\left\{\mathbf{P}_{i, j}\right\}, i=1,2, \ldots, n ; j=1,2, \ldots, m$ with polynomial orders $p$ and $q$ and knots vectors $\Xi=\left\{\xi_{1}, \xi_{2, \ldots . . .} \xi_{n+p+1}\right\}$ and $\mathrm{H}=\left\{\eta_{1}, \eta_{2, \ldots . .} \eta_{n+p+1}\right\}$. Using tensor product a B-Spline surface is obtained.

$$
\mathbf{S}(\xi, \eta)=\sum_{i=1}^{n} \sum_{j=1}^{m} N_{i, p}(\xi) M_{j, q}(\eta) \mathbf{P}_{i, j}
$$

Fig. 3 shows a quadratic B-spline surface obtained by tensor product. 

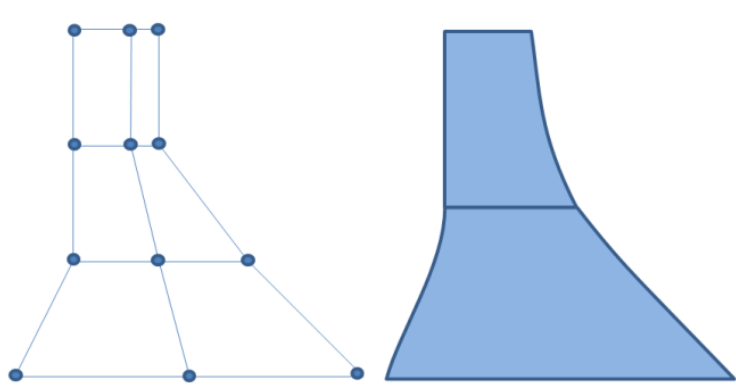

Fig. 3: Quadratic surface with two isogeometric elements and 12 control points.

A NURBS curve can be defined as a parametric rational BSpline curve with a non-uniform knot vector. Equation (5) represents a NURBS curve of $p$ degree, control points $\mathbf{P}_{i}$, with knot vector $\mathbf{\Xi}$ and weights $w$.

$$
\mathbf{C}(\xi)=\frac{\sum_{i=0}^{n} N_{i, p}(\xi) w_{i} \mathbf{P}_{i}}{\sum_{j=0}^{n} N_{j, p}(\xi) w_{j}}
$$

These rational curves enable efficient computational processing and compact storage of data [17]. The use of open knot vectors allows the insertion of degrees of freedom at domain ends. As rational functions, NURBS curves allow the modeling of conic and quadric surfaces. Fig. 4 shows a quadratic NURBS curve obtained this with $\xi=[0,0,0,1,2,2,3,3,3]$ and $w=[1,1,1,1,1,1]$, the multiplicity in knot 2 indicates $\mathrm{C}^{0}$ continuity in the corresponding geometric point.

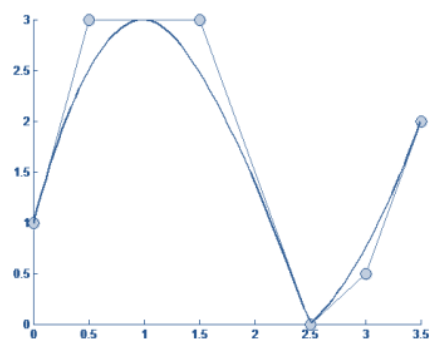

Fig. 4: Quadratic NURBS curve

\section{DYNAMIC RESPONSE OF LINEAR SYSTEMS USING AIG \\ 3.1 Linear elasticity}

Linear elasticity problems can be formulated through the differential equation of equilibrium, this equation in indicial notation became:

$$
\sigma_{i j, j}+f_{i}=0 \quad \text { in } \Omega
$$

Subjected to the following boundary conditions:

$$
\begin{array}{ll}
\sigma_{i j} n_{j}=h_{i} & \text { in } \Gamma_{\sigma} \\
u_{i}=\bar{u} & \text { in } \Gamma_{u}
\end{array}
$$

Equation (7) represents the natural boundary conditions or Neumann conditions and the essential boundary conditions or Dirichlet conditions. The set of Equations (6) and (7), represent the static equilibrium of the body under study.
Boundary conditions represent the support conditions and surface tensions. Generalized Hooke's Law for a linear elastic solid is given by:

$$
\sigma_{i j}=C_{i j k l} \varepsilon_{i j}
$$

The $C_{i j k l}$ tensor contains the elastic coefficients or the constitutive relationship and $\varepsilon_{i j}$ is the infinitesimal deformations tensor. According to [7], the weak form of the linear elasticity problem has the form:

$$
\int_{\Omega} \hat{\sigma}_{i j} w_{i, j} d \Omega=\int_{\Omega} f_{i} w_{i} d \Omega+\int_{\Gamma_{\sigma}} h_{i} w_{i} d \Gamma_{\sigma}
$$

Where $\hat{\sigma}_{i j}$ is an approximate solution of the stress field, $w$ is the weight function that minimizes the residual. Approximating the displacement field with a function like:

$$
\hat{\mathbf{u}}=\sum_{k=1}^{m} N_{k} \mathbf{u}_{k}
$$

In this equation $N_{k}$ are the NURBS basis functions and $\mathbf{u}_{k}$ represents the control point displacements. Applying the Galerkin method Equation (11) is obtained:

$$
\sum_{k=1}^{m}\left(\int_{\Omega} \mathbf{B}^{T} \mathbf{C B} d \Omega\right) \hat{\mathbf{u}}_{k}=\int_{\Omega} \mathbf{N f} d \Omega+\int_{\Gamma_{\sigma}} \mathbf{N h} d \Gamma_{\sigma}
$$

B stands for the NURBS basis functions derivatives matrix, $\mathbf{C}$ is the constitutive matrix, $\mathbf{N}$ is the NURBS basis functions matrix, $\mathbf{f}$ represents the body forces and $\mathbf{h}$ the surface tensions. If $N$ is the number of Degrees of Freedom (DOF), Equation (11) represents the $N$ dimension algebric system to be solved and the system stiffness matrix is:

$$
\mathbf{k}=\sum_{k=1}^{m}\left(\int_{\Omega} \mathbf{B}^{T} \mathbf{C B} d \Omega\right) \hat{\mathbf{u}}_{k}
$$

\subsection{Natural vibration frequencies and modes using IGA}

The generalized eigenvalue problem, whose solution gives the natural frequencies and modes of a system, can be represented in matrix form (characteristic equation):

$$
\left(\mathbf{k}-\boldsymbol{\Omega}^{2} \mathbf{m}\right) \boldsymbol{\Phi}=\mathbf{0}
$$

Where $\Phi$ represent the eigenvector matrix that represents the plane vibration shape modes, $\boldsymbol{\Omega}^{2}$ is the square of the angular frequency $\left(\omega_{n}\right)$. $\mathbf{k}$ is the stiffness matrix described in Equation (12) and $\mathbf{M}$ is the consistent mass matrix calculated by:

$$
\mathbf{m}=\int_{\Omega} \rho \mathbf{N}_{j}^{T} \mathbf{N}_{k} d \Omega
$$

Matrices $\mathbf{N}_{j}$ and $\mathbf{N}_{k}$ are the NURBS basis functions matrices and $\rho$ stands for the material density. The $\mathrm{N}$ roots, $\omega_{n}^{2}$ of Equation (13) determine the $N$ natural frequencies of vibration, arranged in sequence from smallest to largest. When a natural frequency $\omega_{\mathrm{n}}$ is known, the characteristic equation can be solved for the corresponding vector $\phi_{n}$ within a multiplicative constant. The eigenvalue problem gives the shape of the vector given by the relative values of the $N$ displacements $\phi_{j n}(j=1,2, \ldots, N)$. Corresponding to the $N$ natural vibration frequencies of an $N$-DOF system, there are $N$ 
independent vectors $\phi_{n}$, which are known as natural modes of vibration [18].

This eigenvalue problem was successfully solved for plane stress and plane strain states using IGA in [19]. In this work the research continues with the dynamic response for free and forced vibrations for undamped and damped systems.

In order to test the IGA formulation, a Matlab® script was implemented following some of the guidelines described in [20].

\subsection{Free vibration analysis for undamped systems}

The equations of motion for a free linear Multi Degree of Freedom (MDF) system without damping are:

$$
\mathbf{m u ̈}+\mathbf{k u}=\mathbf{0}
$$

Equation (15) represents $N$ homogeneous differential equations that are coupled. The solution satisfies the initial conditions:

$$
\begin{aligned}
\mathbf{u} & =\mathbf{u}(\mathbf{0}) \\
\dot{\mathbf{u}} & =\dot{\mathbf{u}}(\mathbf{0})
\end{aligned}
$$

The natural frequencies and vibration modes are obtained from the eigenvalue problem described in Equation(13). The $N$ eigenvalues and $N$ natural modes can be assembled compactly into matrices.

$$
\begin{gathered}
\boldsymbol{\Phi}=\left[\phi_{n j}\right]=\left[\begin{array}{cccc}
\phi_{11} & \phi_{12} & \cdots & \phi_{1 N} \\
\phi_{21} & \phi_{22} & \cdots & \phi_{1 N} \\
\vdots & \vdots & \ddots & \vdots \\
\phi_{N 1} & \phi_{N 2} & \cdots & \phi_{N N}
\end{array}\right] \\
\mathbf{\Omega}^{2}=\left[\begin{array}{cccc}
\omega_{1}^{2} & 0 & \cdots & 0 \\
0 & \omega_{2}^{2} & \cdots & 0 \\
\vdots & \vdots & \ddots & \vdots \\
0 & 0 & \cdots & \omega_{N}^{2}
\end{array}\right]
\end{gathered}
$$

The $\Phi$ matrix is called modal matrix and $\Omega^{2}$ the spectral matrix for the eigenvalue problem. The natural modes are proven to be orthogonal with respect to $\mathbf{m}$ and $\mathbf{k}$ matrices, see e.g. [21], so the following matrices are diagonal:

$$
\begin{array}{r}
\mathbf{K}=\boldsymbol{\Phi}^{T} \mathbf{k} \boldsymbol{\Phi} \\
\mathbf{M}=\boldsymbol{\Phi}^{T} \mathbf{m} \boldsymbol{\Phi}
\end{array}
$$

Each term in the diagonal of $\mathbf{K}$ and $\mathbf{M}$ is denoted by $K_{n}$ and $M_{\mathrm{n}}$ respectively. Natural modes vectors can be used as a basis to represent any displacement vector $\mathbf{u}$ :

$$
\mathbf{u}=\sum_{r=1}^{N} \phi_{r} q_{r}=\mathbf{\Phi q}
$$

Where $q_{r}$ are scalar multipliers called modal coordinates and $\mathbf{q}$ can be calculated by:

$$
q_{n}=\frac{\phi_{n}^{T} \mathbf{m u}}{M_{n}}
$$

The solution for Equation (15) in modal coordinates is given by [18]:

$$
\mathbf{u}(t)=\sum_{n=1}^{N} \phi_{n}\left[q_{n}(0) \cos \omega_{n} t+\frac{\dot{q}_{n}(0)}{\omega_{n}} \sin \omega_{n} t\right]
$$

Or

$$
u(t)=\sum_{n=1}^{N} \phi_{n} q_{n}(t)
$$

Where

$$
q_{n}(t)=q_{n}(0) \cos \omega_{n} t+\frac{\dot{q}_{n}(0)}{\omega_{n}} \sin \omega_{n} t
$$

Equation (23) represents the displacement $\mathbf{u}$ as a function of time and the initial displacement and velocity conditions. By solving the eigenvalue problem, the natural frequencies $\omega_{n}$ and modes $\phi_{n}$ are already known so the displacement response can be determined. The response in velocity and acceleration can be obtained analytically or numerically from these expressions.

\subsubsection{Undamped free vibrations: Concrete gravity dam section}

The results obtained for a plane strain state problem are showed. This solution aims to determine the dynamic response in displacements, velocity and acceleration for a dam section. Plane strain state is defined when the strain state at a material point is such that the only non-zero strain components act in one plane only. This hypothesis can be adopted for the analysis of a cross section of a dam, where the cross-section analyzed is contained within the dam mass [22]. The problem consists to study a generic section of a gravity concrete dam whose dimensions are shown in Fig. 5(a). Considering the base as fixed and the physical properties of the material are defined by elasticity modulus $E=21 G P a, v=$ 0.2 and $\rho=25 \mathrm{kN} / \mathrm{m}^{3}$. The initial conditions are $u_{i}(0)=1 \mathrm{~m}$ and $\dot{\mathbf{u}}(0)=0$, where $u_{i}$ stands for the DOF's on top of the dam. In this problem 16 quadratic isogeometric elements were used, as illustrated in Fig. 5(b).

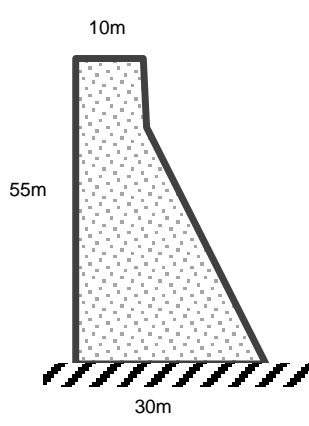

(a)

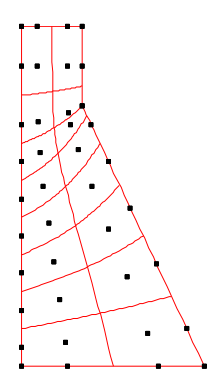

(b)
Fig. 5: Cross section of a concrete gravity dam, discretization and initial conditions.

The numerical response using the procedures described above, are represented in Fig. 6, where the displacements $\mathbf{u}(t)$, velocity $\dot{\mathbf{u}}(t)$ and acceleration $\ddot{\mathbf{u}}(t)$ are showed. 

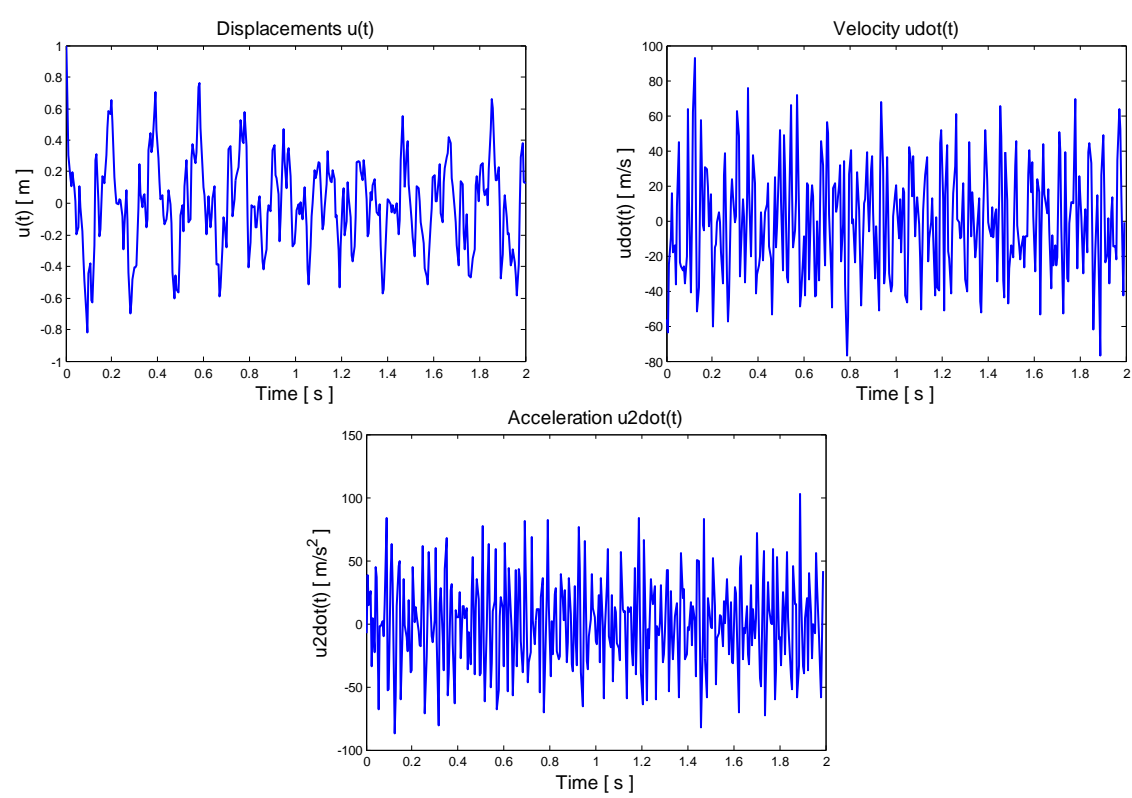

Fig. 6: Free undamped dynamic response for the dam cross section $\mathbf{u}(t), \dot{\mathbf{u}}(t)$ and $\ddot{\mathbf{u}}(t)$.

\subsection{Free vibration analysis for systems with classical damping}

In this case the differential equation that governs the problem is:

$$
\mathbf{m u ̈}+\mathbf{c u}+\mathbf{k u}=\mathbf{0}
$$

Where $\mathbf{c}$ is the damping matrix, if classical damping is considered, the following identity is satisfied by $\mathbf{c}$ [21]:

$$
\mathbf{c m}^{-1} \mathbf{k}=\mathbf{k m}^{-1} \mathbf{c}
$$

Classical damping implies that the damped system have classical natural modes and applying modal transformation in c, gives a diagonal matrix:

$$
\mathbf{C}=\boldsymbol{\Phi}^{T} \mathbf{c} \Phi
$$

Thus there are $N$ uncoupled differential equations in Equation (26) for each natural mode:

$$
M_{n} \ddot{q}_{n}+C_{n} \dot{q}_{n}+K_{n} q_{n}=0
$$

The damping ratio can be defined for each mode of vibration:

$$
\zeta_{n}=\frac{C_{n}}{2 M_{n} \omega_{n}}
$$

Dividing Equation (29) by $M_{n}$ :

$$
\ddot{q}_{n}+2 \zeta_{n} \omega_{n}^{2} \dot{q}_{n}+\omega_{n}^{2} q_{n}=0
$$

The solution of Equation (31) is known [18], [21]:

$$
q_{n}(t)=e^{-\zeta_{n} \omega_{n} t}\left[q_{n}(0) \cos \omega_{n D} t+\frac{\dot{q}_{n}(0)+\zeta_{n} \omega_{n} q_{n}(0)}{\omega_{n D}} \sin \omega_{n D} t\right]
$$

Where:

$$
\omega_{n D}=\omega_{n} \sqrt{1-\zeta_{n}^{2}}
$$

And therefore:

$$
\mathbf{u}(t)=\sum_{n=1}^{N} \phi_{n} e^{-\zeta_{n} \omega_{n} t}\left[q_{n}(0) \cos \omega_{n D} t+\frac{\dot{q}_{n}(0)+\zeta_{n} \omega_{n} q_{n}(0)}{\omega_{n D}} \sin \omega_{n D} t\right]
$$

The latter gives the solution for the free vibration problem with classic damping. The displacements are function of time and the initial conditions. Velocity and acceleration can be calculated analytically or by numerical differentiation.

3.4.1 Concrete gravity dam section: free vibrations with damping

Using the same of data of the last example and a damping ratio $\zeta=5 \%$, recommended value in [23], the dynamic response obtained is illustrated in Fig. 7.

Damping ratio in this analysis correctly shows the decay of movement, the system oscillates with amplitude decreasing with every cycle of vibration. This kind of motion is typical of underdamped systems. 

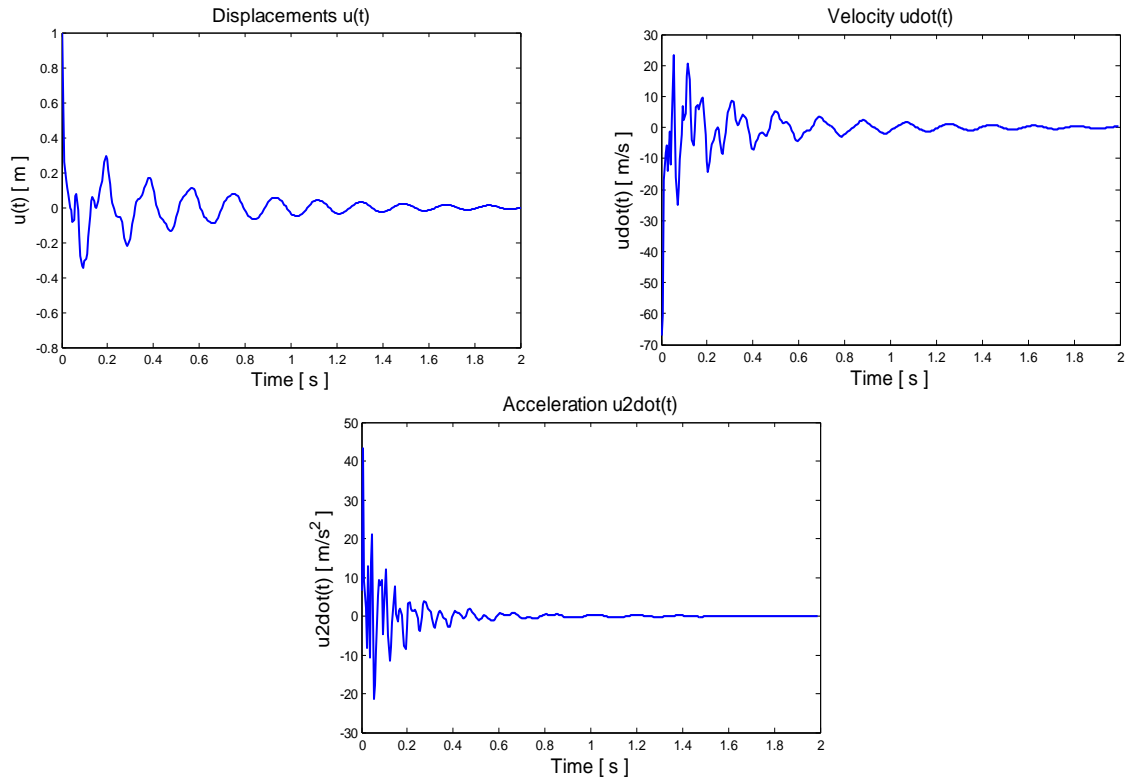

Fig. 7: Dynamic response $\mathbf{u}(t), \dot{\mathbf{u}}(t)$ and $\ddot{\mathbf{u}}(t)$ for free damped vibrations.

\subsection{Forced vibrations analysis with AIG}

Forced vibrations can be modelled as:

$$
\mathbf{m} \ddot{\mathbf{u}}+\mathbf{c} \dot{\mathbf{u}}+\mathbf{k} \mathbf{u}=\mathbf{p}(t)
$$

Where $\mathbf{p}(t)$ represents an excitation in the system that is time depending. After using the modal transformation in Equations (19), (20), (21) and (28) an equivalent problem is stated:

$$
\mathbf{M} \ddot{\mathbf{q}}+\mathbf{C} \dot{\mathbf{q}}+\mathbf{K q}=\mathbf{P}(t) \text { with } \mathbf{P}(t)=\boldsymbol{\Phi}^{T} \mathbf{p}(t)
$$

For the solution of this kind of problem is possible to adopt a time-step method such as the Central Difference Method (CDM). Knowing the natural frequencies and vibration modes is possible to apply any of those methods. In this work CDM is used to obtain the dynamic response. For damped systems the Rayleigh damping [21] was constructed specifying the damping ratio $\zeta_{n}$ for the first and sixth natural modes. The algorithm used is depicted in Table 1.

\section{Table 1: Central difference method algorithm [18]}

I Preliminary calculation

$$
\begin{aligned}
& \text { 1. } \mathbf{q}_{0}=\left(\boldsymbol{\Phi}^{T} \mathbf{m} \boldsymbol{\Phi}\right)^{-1} \cdot\left(\boldsymbol{\Phi}^{T} \mathbf{m} \mathbf{u}_{0}\right) ; \quad \dot{\mathbf{q}}_{0}=\left(\boldsymbol{\Phi}^{T} \mathbf{m} \boldsymbol{\Phi}\right)^{-1} \cdot\left(\boldsymbol{\Phi}^{T} \mathbf{m} \dot{\mathbf{u}}_{0}\right) \\
& \text { 2. } \mathbf{P}_{0}=\boldsymbol{\Phi}^{T} \mathbf{p}_{0} \\
& \text { 3. } \ddot{\mathbf{q}}_{0}=\mathbf{M}^{-1}\left(\mathbf{P}_{0}-\mathbf{C} \dot{\mathbf{q}}_{0}-\mathbf{K} \mathbf{q}_{0}\right) \\
& \text { 4. } \mathbf{q}_{-1}=\mathbf{q}_{0}-\Delta t \dot{\mathbf{q}}_{0}+\frac{\Delta t^{2}}{2} \ddot{\mathbf{q}}_{0} \\
& \text { 5. } \hat{\mathbf{K}}=\frac{1}{\Delta t^{2}} \mathbf{M}+\frac{1}{2 \Delta t} \mathbf{C} \\
& \text { 6. } \mathbf{a}=\frac{1}{\Delta t^{2}} \mathbf{M}-\frac{1}{2 \Delta t} \mathbf{C} \\
& \text { 7. } \mathbf{b}=\mathbf{K}-\frac{2}{\Delta t^{2}} \mathbf{M}
\end{aligned}
$$

II Calculation in each $\Delta t$

$$
\begin{aligned}
& \text { 1. } \mathbf{P}_{i}=\boldsymbol{\Phi}^{T} \mathbf{p}_{i} \\
& \text { 2. } \hat{\mathbf{P}}_{i}=\mathbf{P}_{i}-\mathbf{a q}_{i-1}-\mathbf{b} \mathbf{q}_{i} \\
& \text { 3. } \mathbf{q}_{i+1}=\hat{\mathbf{K}}^{-1} \hat{\mathbf{P}}_{i} \\
& \text { 4. } \mathbf{u}_{i+1}=\boldsymbol{\Phi} \mathbf{q}_{i+1}
\end{aligned}
$$

3.5.1.1 Concrete gravity dam section: forced vibrations with damping

In this numerical example an harmonic excitation $p(t)=p_{0} \sin \left(\omega_{e} t\right)$ is applied on top of the dam as showed in Fig. 8.

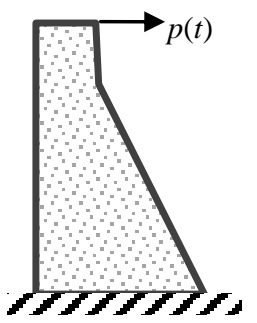

Fig. 8 : Harmonic excitation on dam section

For $p_{0}=80 \mathrm{kN}$ and $\omega_{e}$ equal to $0.5 \omega_{1}, 1.0 \omega_{1}$ and $2.0 \omega_{1}$, where $\omega_{1}=$ first natural frequency, Fig. 9. Shows the dynamic response and in Fig. 9 (a) it's possible to identify the transient vibration at the beginning of the graphics which depends of initial conditions then it shows the characteristic of the forced vibration or steady-state vibration. This behavior is expected when the frequency of external excitation is not closer to a natural frequency of vibration.

Fig. 9 (b) shows the excitation approaching the resonant frequency, the displacement amplitude increasing with every cycle of vibration. The growth of the amplitude is limited because of damping. 

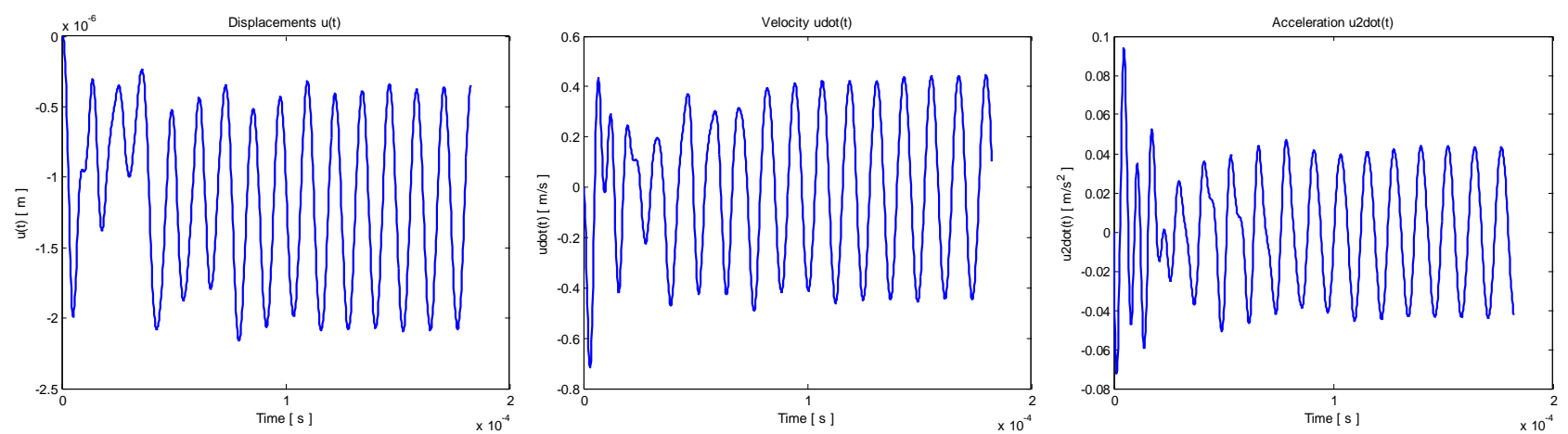

(a)
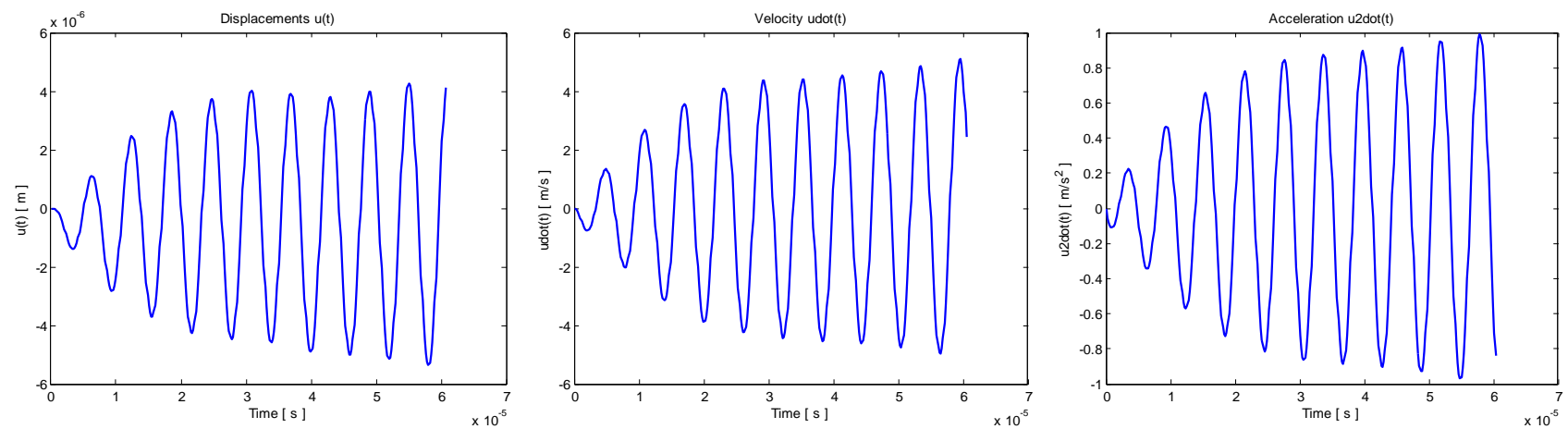

(b)

Fig. 9: Dynamic response for forced vibrations (a) $\omega_{e}$ equal to $0.5 \omega_{1}$ (b) $\omega_{e}$ equal to $\omega_{1}$

\section{CONCLUSIONS}

Previous studies of eigenvalue problems showed satisfactory performance of NURBS as approximation functions [19]. The results in this study are indicatives that IGA can be successfully applied in transient analysis for dam models.

The dynamic responses obtained showed agreement with theory, the resonance effect and the steady state components of movement were effectively identified. These capabilities together with IGA open many possibilities for vibration studies in dams.

Future works may include more complex excitations as seismic analysis together with other time-step methods such the Newmark methods.

\section{ACKNOWLEDGMENTS}

The support of the Technological Park of Itaipu (PTI) and the Center for Advanced Studies in Dam Safety (CEASB) are gratefully acknowledged.

\section{REFERENCES}

[1] C. F. Corns, G. S. Tarbox, and E. K. Schrader, "Gravity dam design and analysis," in Advanced dam engineering for design, construction, and rehabilitation, New York: Van Nostrand Reinhold, 1988, pp. 466-492.

[2] D. Balageas, C.-P. Fritzen, and A. Gemes, Eds., Structural Health Monitoring. London, UK: ISTE, 2006.

[3] C. R. Farrar and K. Worden, "An introduction to structural health monitoring," Philos. Trans. R. Soc. A Math. Phys. Eng. Sci., vol. 365, no. 1851, pp. 303-315, Feb. 2007.

[4] A. K. Chopra, "Earthquake resistant design of concrete gravity dams," J. Struct. Div., vol. 104, no. 6, pp. 953-
$971,1978$.

[5] K. J. Bathe, Finite element procedures. New Jersey: Prentice Hall, 2006.

[6] T. J. R. Hughes, The Finite Element Method. Mineola, NY: Dover, 2000.

[7] J. N. Reddy, An Introduction to the Finite Element Method. Highstown: McGraw - Hill, 1993.

[8] J. A. Cottrell, T. J. R. Hughes, and Y. Bazilevs, Isogeometric Analysis: Toward Integration of CAD and FEA, 1st ed. Chichester: John Wiley \& Sons, 2009.

[9] T. J. R. Hughes, J. A. Cottrell, and Y. Bazilevs, "Isogeometric analysis: CAD, finite elements, NURBS, exact geometry and mesh refinement," Comput. Methods Appl. Mech. Eng., vol. 194, no. 39-41, pp. 4135-4195, 2005.

[10] J. A. Cottrell, A. Reali, Y. Bazilevs, and T. J. R. Hughes, "Isogeometric analysis of structural vibrations," Comput. Methods Appl. Mech. Eng., vol. 195, no. 41-43, pp. 5257-5296, 2006.

[11] CEASB, Acervo técnico do CEASB. Itaipu Binacional: Centro de Estudos Avançados em Segurança de Barragens, 2015.

[12] Y. Bazilevs, V. M. Calo, J. A. Cottrell, T. J. R. Hughes, A. Reali, and G. Scovazzi, "Variational multiscale residual-based turbulence modeling for large eddy simulation of incompressible flows," Comput. Methods Appl. Mech. Eng., vol. 197, no. 1-4, pp. 173-201, 2007.

[13] D. J. Benson, Y. Bazilevs, M. C. Hsu, and T. J. R. Hughes, "Isogeometric shell analysis: The Reissner- 
Mindlin shell," Comput. Methods Appl. Mech. Eng., vol. 199, no. 5-8, pp. 276-289, 2010.

[14] J. Kiendl, R. Schmidt, R. Wüchner, and K.-U. Bletzinger, "Isogeometric shape optimization of shells using semi-analytical sensitivity analysis and sensitivity weighting," Comput. Methods Appl. Mech. Eng., vol. 274, pp. 148-167, 2014.

[15] S. Cho and S. H. Ha, "Isogeometric shape design optimization: Exact geometry and enhanced sensitivity," Struct. Multidiscip. Optim., vol. 38, no. 1, pp. 53-70, 2009.

[16] J. A. Cottrell, T. J. R. Hughes, and A. Reali, "Studies of refinement and continuity in isogeometric structural analysis," Comput. Methods Appl. Mech. Eng., vol. 196, no. 41-44, pp. 4160-4183, 2007.

[17] L. Piegl and W. Tiller, The NURBS book. Berlin: Springer Verlag, 1997.

[18] A. K. Chopra, Dynamics of structures: theory and applications to earthquake engineering. Upper Saddle River, NJ:. Pearson/Prentice Hall, 2012.

[19] R. J. Mendoza Fakhye, R. D. Machado, M. Rauen, and M. Arndt, "Free vibration analysis of gravity dam sections using isogeometric analysis," Int. J. Eng. Appl. Sci., vol. 8, no. 4, pp. 1-9, 2016.

[20] V. P. Nguyen, C. Anitescu, S. P. A. A. Bordas, and T. Rabczuk, "Isogeometric analysis: An overview and computer implementation aspects," Math. Comput. Simul., vol. 117, pp. 1-114, 2015.

[21] R. W. Clough and J. Penzien, Dynamics of structures. Berkeley, CA: Computers \& Structures Inc., 1995.

[22] R. B. Jansen, Advanced dam engineering for design, construction, and rehabilitation. Springer Science \& Business Media, 2012.

[23] FEMA, Federal Guidelines for Dam Safety. USA: Federal Emergency Management Agency, 2005 